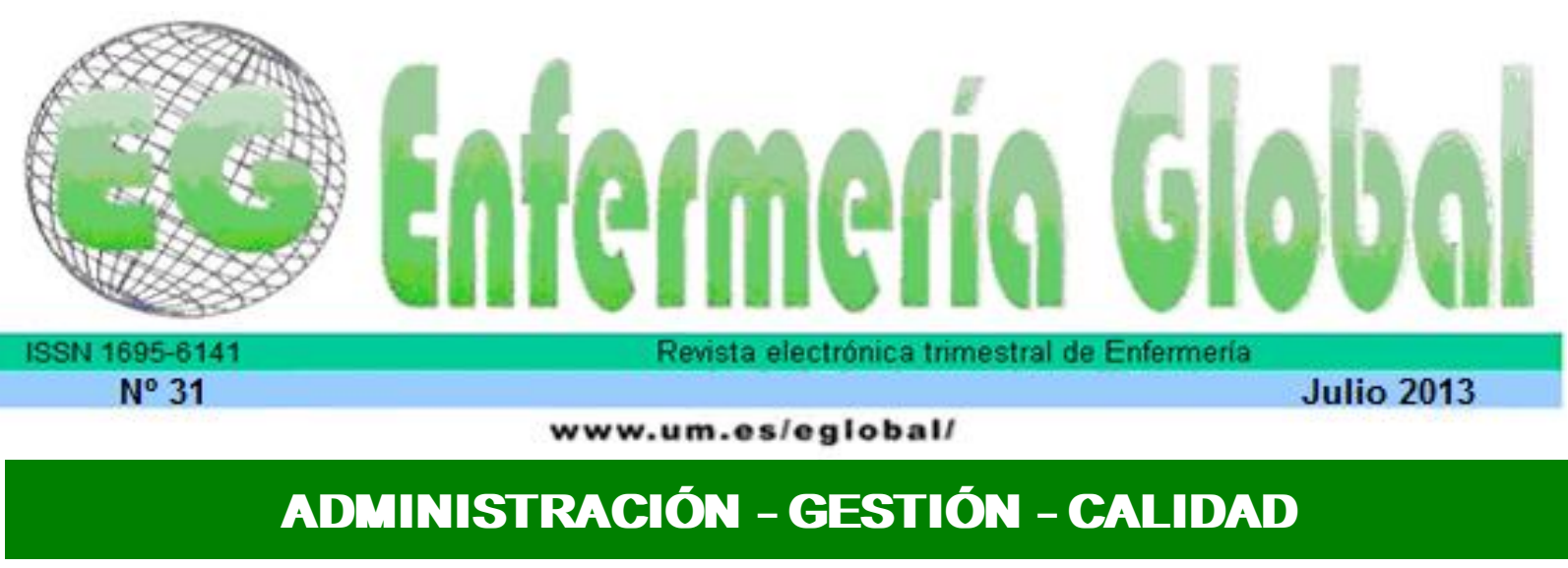

\title{
Valoración de la satisfacción de usuarios de consulta de Enfermería en Centros de Salud a partir de indicadores de calidad técnicos y de comunicación
}

Evaluation of user satisfaction of nursing consultation in health centers based on quality and technical communication indicators

\section{"Dios Guerra, Caridad ${ }^{* *}$ Alba Dios, $\mathrm{M}^{\mathrm{a}}$ Antonia ${ }^{* * *}$ Ruiz Moral, Roger ****Jiménez García, Celia *****Pérula de Torres, Luis Ángel ${ }^{* * * * * *}$ Rodríguez Borrego, $\mathrm{M}^{\mathrm{a}}$ Aurora}

*Enfermera. Centro de Salud de Azahara-Occidente. Distrito Córdoba. E-mail: caridad.dios.sspa@juntadeandalucia.es **Psicóloga. Unidad Docente de Medicina de Familia y Comunitaria de Córdoba. ***Médico de Familia. Jefe de la Unidad Docente de Medicina de Familia y Comunitaria de Córdoba. Facultad de Medicina. ${ }^{* * * *}$ Médico preventivista. Epidemióloga del Distrito Sanitario Guadalquivir, Córdoba. ${ }^{* * * * *}$ Médico preventivista. Técnico de Salud de la Unidad Docente de Medicina de Familia y Comunitaria de Córdoba. Facultad de Medicina. ${ }^{* * * * * *}$ Directora Departamento de Enfermería. Universidad de Córdoba.

Este trabajo ha sido financiado por la Consejería de Salud de la Junta de Andalucía (Ayudas a proyectos de investigación, Expte.: PI0103/2008).

Palabras clave: Satisfacción; cualitativo; relación; información; tiempo; procedimiento.

Keywords: Satisfaction; qualitative; relationship; information; time; procedure.

\section{RESUMEN}

Objetivos: Medir la satisfacción relacionada con aspectos técnicos y comunicativos de los usuarios de consulta de Enfermería en Atención Primaria.

Métodos: Estudio descriptivo realizado con pacientes usuarios del Servicio de Enfermería de los Centros de Salud (CCSS) de Córdoba y provincia. Los pacientes fueron entrevistados utilizando dos tipos de preguntas, cerradas y abiertas que recogían sus opiniones y satisfacción en dominios comunicacionales como la relación enfermera-paciente, la información recibida o el tiempo empleado; así como los procedimientos utilizados. Análisis descriptivo e inferencial (Test de Ji-cuadrado; $p<0,05$ ). Las preguntas abiertas se agruparon en categorías en un proceso de análisis cualitativo que implicó a dos investigadoras independientes. 
Resultados: Participaron 335 pacientes. Se obtuvo un $76,5 \%$ de satisfacción en las respuestas cerradas respecto a los procedimientos utilizados en la sala de curas, sin embargo el $26,1 \%$ de los pacientes planteo alguna sugerencia o propuesta de cambio en las respuestas abiertas. Al $89,1 \%$ de satisfacción en información recibida en respuestas cerradas, el 16,1\% hizo sugerencias de cambio en las respuestas abiertas. En cuanto a la relación enfermera paciente, los resultados fueron: un 94,2\% mostraba satisfacción frente a un 7,5\% que planteó sugerencias en preguntas abiertas; en referencia al tiempo empleado la satisfacción oscilo del $88,5 \%$ al $16,8 \%$.

Conclusiones: La mayoría de los pacientes que consultaron con Enfermería en los Centros de Salud mostraron satisfacción con la atención recibida, si bien les gustaría participar más en la toma de decisiones.

\section{ABSTRACT}

Objectives: To measure the satisfaction related to communication and technical aspects of the users of nursing consultation in Primary Care.

Material and methods: Descriptive study conducted with patients using the nursing service Health Centers. After consultation with the staff, the patients were interviewed using two types of open and closed questions that assessed their views and satisfaction with some communication domains. Frequency distributions and exploring the differences between the two measures were explored using descriptive and inferential analysis (chi-square test, $P<0.05$ ). Open-ended questions were grouped into categories in a process of qualitative analysis involving two researchers independently.

Results: 335 patients participated, $76.5 \%$ was obtained of the closed-ended satisfaction regarding the procedures used in the treatment room, and however, $26.1 \%$ of patients won't have any suggestions or proposed changes in the open responses. To $89.1 \%$ of satisfaction with information received in closed responses, $16.1 \%$ made suggestions for change in the open answers. As the nurse patient, the results were $94.2 \%$ showed satisfaction, compared to $7.5 \%$ which raised suggestions on open questions, and time used the ratio was $88.5 \%$ vs. $16.8 \%$.

Discussion and Conclusions: Most patients who consult with nurses in health centers showed satisfaction with care received, however they would like to participate more in decision making.

\section{INTRODUCCIÓN}

Desde Atención Primaria, y sobre todo desde las consultas de Enfermería se quiere dar al usuario protagonismo en el cuidado de su salud, responsabilizándolo en actuaciones de promoción, prevención y autocuidados e involucrándolo en intervenciones de Participación Ciudadana ${ }^{(1)}$.

La implicación del ciudadano en política sanitaria lleva indiscutiblemente un conocimiento amplio de cómo funciona el sistema sanitario y sus profesionales. Actualmente nos encontramos con usuarios más exigentes, con más conocimientos sanitarios, que demandan mejoras sanitarias que ayuden a aumentar su nivel de satisfacción; en este proceso de implicación el sistema sanitario valora cada vez más la opinión de los pacientes y la calidad de los servicios prestados.

La satisfacción de los usuarios se constituye como uno de los componentes más importantes a la hora de medir la calidad de los servicios sanitarios ${ }^{(2)}$, tal y como afirma Donabedian ${ }^{(3)}$ es imposible describir correctamente la calidad sin contar con el punto de vista del cliente, ya que su opinión nos proporciona información acerca del éxito o fracaso del sistema sanitario para cubrir sus expectativas ${ }^{(4,5)}$. 
La adopción de la perspectiva del cliente, al definir la calidad del servicio, introduce el concepto de percepción, que supone considerar que el usuario del sistema es el único que puede determinar si un servicio es de calidad o no ${ }^{(6)}$.

Un elemento fundamental en la evaluación de los servicios sanitarios es la valoración de sus profesionales, tanto desde el punto de vista técnico, como a nivel relacional y trato con el usuario ${ }^{(7)}$. Entre estos profesionales se encuentran las enfermeras. Hay estudios que revelan que la buena comunicación, la información, la empatía, la apariencia en los cuidados y la capacidad técnica de estos profesionales son fuertes predictores de la evaluación del cuidado recibido ${ }^{(8)}$.

Son varios los estudios que abordan el tema de la percepción y satisfacción de la población con los diferentes servicios sanitarios en los que se han utilizado indistintamente metodologías cuantitativas y cualitativas, tanto de Atención Primaria (1,3,9-10), como en servicios hospitalarios ${ }^{(5,8,11-15)}$, servicios de urgencias $^{(16-18)}$ y con otros profesionales de la salud ${ }^{(19)}$. También, recientes resultados en estudios de medidas directas de satisfacción demuestran que la mayoría de las respuestas de los pacientes/usuarios tienden a inclinarse de forma contundente hacia las opciones de "satisfecho" y "muy satisfecho", siendo en este caso los médicos (92,5\%), los profesionales mejor valorados, seguidos por los profesionales de enfermería $(80,7 \%)$ (20)

Con la intención de mejorar el conocimiento al respecto nos planteamos en este trabajo, como objetivo general, conocer la satisfacción directa de los usuarios con la relación enfermera-paciente, con la información que el usuario recibe de la enfermera, con el tiempo empleado en la consulta de enfermería, y con el procedimiento técnico empleado por la enfermera en sus intervenciones terapéuticas.

\section{MATERIAL Y MÉTODOS}

Diseño observacional descriptivo en pacientes que acudieron a centros de salud urbanos y rurales de Andalucía con la finalidad de recibir atención profesional en consultas programadas de enfermería (procesos: diabetes, hipertensión arterial, obesidad, control del niño sano, embarazo y planificación familiar) y en la sala de curas.

El número de usuarios incluidos se calculó para la estimación de una proporción poblacional, para una $\mathrm{p}=87,4 \%$ (resultado de la encuesta de satisfacción del SAS, 2007), un error alfa del 5\% (95\% de seguridad) y una precisión de $\pm 5 \%$ (margen de error), estimándose en 340 el número de usuarios a reclutar. Se seleccionaron al azar 9 Centros de Salud (CCSS) de diferentes distritos de Atención Primaria, 6 urbanos y 3 rurales. Los pacientes fueron seleccionados mediante muestreo consecutivo en la sala de curas y de consultas programadas de enfermería. Las entrevistas fueron realizadas por dos encuestadores adiestrados para este fin. En cada uno de los CCSS se recogieron las opiniones de los pacientes atendidos por un número mínimo de 3 profesionales al objeto de asegurar variabilidad suficiente en cada centro. Finalmente se obtuvieron encuestas de pacientes atendidos por entre 5-9 enfermera/os en cada uno de los 9 Centros seleccionados ( 55 enfermera/os en total). La media de pacientes entrevistados fue de 37 por Centro y de 6 encuestas por cada enfermera/o.

Se diseñó un cuestionario (Anexo I) adaptado, que exploraba diferentes dominios comunicativos relacionados con: la relación enfermera-paciente, la información 
recibida, el tiempo empleado, los procedimientos en la sala de curas y toma de decisiones en la consulta de Enfermería. Mediante pregunta cerrada, con cuatro opciones de respuesta, se le preguntaba al paciente su opinión sobre el grado de satisfacción en los diferentes dominios explorados. Una pregunta abierta recogía los posibles deseos de cambio respecto a la participación. El cuestionario, seleccionado por conveniencia, fue la readaptación de otro utilizado por Levy Storms, L et al, ${ }^{(21,22)}$ para valorar la satisfacción de ancianos residentes en centros geriátricos. Se comprobó previamente su validez de consenso y aparente, posteriormente fue objeto de un pilotaje con 60 usuarios procedentes de dos Centros de Salud, uno rural y otro urbano, donde se verificó la viabilidad del sistema organizativo y de recogida de información establecido, y se revisó asegurándose de que la redacción de las preguntas era entendida por los pacientes. Tras el pilotaje se realizaron modificaciones organizativas y estructurales para el correcto desarrollo del trabajo de campo.

Los resultados fueron objeto de un análisis cuantitativo de tipo descriptivo utilizando medidas de centralización, dispersión y posición en las variables cuantitativas; medidas de frecuencia absoluta y relativa en las cualitativas, e intervalos de confianza para el $95 \%$ de seguridad $-I C 95 \%$. Se usaron contrastes bilaterales, para un valor de $p \leq 0,05$. Los datos han sido analizados con los programas SPSS (versión 15.0 para Windows) y EPIDAT (versión 3.1).

La existencia de posibles diferencias entre las medidas obtenidas entre las preguntas cerradas y las abiertas se exploró mediante la prueba Ji Cuadrática de Pearson. El análisis principal de las preguntas abiertas fue de tipo cualitativo y se hizo mediante un proceso de categorización de las respuestas, hecho de forma independiente por dos investigadoras. Posteriormente se realizó un proceso de triangulación de datos que se llevo a cabo durante dos fases, la primera para contrastar los análisis de cada investigadora, y la segunda para poner en común la categorización de las respuestas aportadas. Las discrepancias se resolvieron por consenso. Finalmente se obtuvo un conjunto de categorías y subcategorías comunes que agruparon a todas las respuestas obtenidas.

\section{RESULTADOS}

Se incluyeron 335 usuarios: 69,3 \% (232) mujeres (IC95\%: 64,2-74,3\%), con rango de edad de 19 a 89 años y edad media de 55,2 años $\pm 18,0$ (DT) años (IC95\%: 53,357,1). El 65,4 \%(IC95\%: 60,1-70,6\%) estaban casados, la mayoría tenían estudios primarios (40\%; IC95\%: 34,6-45,4\%) o no tenían estudios (25,1\%; IC95\%: 20,3$29,9 \%)$. Los principales motivos por los que acudieron a consulta de enfermería fueron para curas (24,2\%; IC95\%: $19,4-28,9 \%)$ y para consultas programadas $(74,3 \%$; IC95\%: 69,5-79,2\%;). En consultas programadas, un 36,4\% acudieron para control de diabetes, el $53,1 \%$ para control de tensión arterial, el $41,2 \%$ para obesidad y el $7,8 \%$ para control del embarazo. Otros motivos por los que los pacientes acudieron a la consulta de enfermería fueron control del niño sano, colesterol, ECG, espirometrías y vacunaciones.

En la tabla 1 se especifica el número de respuestas obtenidas en las diferentes categorías sobre satisfacción en los cuatro dominios comunicacionales, así como el número de comentarios de los pacientes que aportaron propuestas de cambio al respecto. En la tabla 2 se exponen los resultados del análisis cualitativo sintetizándose el grupo de categorías y subcategorías identificadas. 
Tabla 1. Satisfacción de los usuarios de consultas de Enfermería en los Centros de Salud.

\begin{tabular}{|c|c|c|c|c|c|c|}
\hline \multirow[b]{2}{*}{ Dominio } & \multirow[b]{2}{*}{$\begin{array}{l}\text { Pregunta } \\
\text { a } \\
\text { propuest } \\
\text { as de } \\
\text { cambio }\end{array}$} & \multicolumn{4}{|c|}{ Nivel de Satisfacción } & \multirow[b]{2}{*}{$\begin{array}{c}\text { Valor de } \\
\mathbf{p}^{*}\end{array}$} \\
\hline & & $\begin{array}{c}\text { Muy } \\
\text { satisfecho } \\
\text { n }(\%)\end{array}$ & $\begin{array}{c}\text { Satisfech } \\
\text { o }\end{array}$ & $\begin{array}{c}\text { Insatisfecho } \\
\text { o } \\
\text { regularment } \\
\text { e satisfecho } \\
\text { n }(\%) \\
\end{array}$ & Total & \\
\hline \multirow[t]{2}{*}{ Relación } & $\mathrm{Si}$ & $5(2,8)$ & $13(8,8)$ & $7(100)$ & $25(7,5)$ & \multirow{2}{*}{$<0,0001$} \\
\hline & No & $175(97,2)$ & $135(91,2)$ & $0(0)$ & $310(92,5)$ & \\
\hline \multirow[t]{2}{*}{ Información } & $\mathrm{Si}$ & $9(7)$ & $27(14,8)$ & $17(89,5)$ & $53(16,1)$ & \multirow[t]{2}{*}{$<0,0001$} \\
\hline & No & $120(93)$ & $155(85,2)$ & $2(10,5)$ & $277(83,9)$ & \\
\hline \multirow{2}{*}{$\begin{array}{l}\text { Tiempo } \\
\text { dedicado }\end{array}$} & $\mathrm{Si}$ & $8(6,8)$ & $33(16,5)$ & $15(93,8)$ & $56(16,8)$ & \multirow[t]{2}{*}{$<0,0001$} \\
\hline & No & $110(93,2)$ & $167(83,5)$ & $1(6,3)$ & $278(83,2)$ & \\
\hline \multirow{2}{*}{$\begin{array}{l}\text { Procedimiento } \\
\text { S }\end{array}$} & $\mathrm{Si}$ & $7(18,4)$ & $14(28,6)$ & $3(75)$ & $24(26,4)$ & \multirow[t]{2}{*}{$<0,0001$} \\
\hline & No & $31(81,6)$ & $35(71,4)$ & $1(25)$ & $67(73,6)$ & \\
\hline
\end{tabular}

* Test de la Ji-cuadrado de Pearson

Tabla 2. Sugerencias de los pacientes para la mejora de la calidad de atención en la Consulta de Enfermería

\begin{tabular}{|c|c|c|}
\hline Categorías & \multicolumn{2}{|c|}{ Subcategorías } \\
\hline \multirow[t]{5}{*}{ Mejora de recursos } & \multicolumn{2}{|l|}{ Instalaciones } \\
\hline & \multicolumn{2}{|c|}{ Espacios íntimos } \\
\hline & \multicolumn{2}{|c|}{ Más personal cualificado } \\
\hline & \multicolumn{2}{|l|}{ Más tiempo } \\
\hline & \multicolumn{2}{|c|}{ Menos cambios de personal } \\
\hline \multirow[t]{10}{*}{ Habilidades de comunicación } & Trato & Trato amable cercanía \\
\hline & & Trato personal, implicación \\
\hline & Información & Cantidad de información \\
\hline & & Calidad de información \\
\hline & & Tipo: Oral/Escrita \\
\hline & \multicolumn{2}{|c|}{ Escucha activa } \\
\hline & \multicolumn{2}{|l|}{ Interés } \\
\hline & \multicolumn{2}{|c|}{ Disponibilidad } \\
\hline & \multicolumn{2}{|l|}{ Empatía } \\
\hline & \multicolumn{2}{|c|}{ Dejar hablar al paciente } \\
\hline \multirow[t]{3}{*}{ Tiempo de atención } & \multicolumn{2}{|l|}{ Más tiempo } \\
\hline & \multicolumn{2}{|l|}{ De calidad } \\
\hline & \multicolumn{2}{|c|}{ Demora en las consulta } \\
\hline
\end{tabular}


Se observa que los comentarios más representativos de los pacientes aluden al dominio referido al procedimiento en la sala de curas y sus opiniones inciden en la necesidad de mejorar los recursos del centro, en aspectos tales como el espacio para lograr una mayor intimidad, mejorar las instalaciones, ampliar el número de profesionales que atienden y su cualificación, evitando los cambios de personal, también se hace referencia a que el tiempo empleado en la cura sea mayor, a la mejora del trato y de la atención al paciente:

"Como no conozco el protocolo...las instalaciones deberían estar mejor. Estaban dos personas en la sala de curas y, aunque había un biombo, más intimidad. Dotar al profesional de más medios y mejor espacio."

"Que se pare más cuando un paciente necesite más tiempo y no cambiar tanto a los enfermeros ya que tanto cambio es un problema, pues tengo que explicarle mis problemas cada vez que cambien de profesional es muy incómodo"

"Que atiendan mejor a los enfermos, que atiendan más tiempo y que lo haga mejor. Me lo hizo muy mal, a los 5 minutos ya tenía la venda por los suelos".

Por otra parte también existen categorías que son comunes a los 4 dominios comunicacionales. En varios de los comentarios de estas categorías se manifiesta la necesidad de los pacientes de recibir una atención y un trato, cercano, humano, cálido, paciente...

"El que estudia una carrera de estas tiene que ser humano"

"Que se dejara de cháchara y atendiera al enfermo. Se puso a hablar con los compañeros de tonterías mientras yo esperaba a que me curara".

Varios comentarios hacen alusión a la necesidad de ser escuchados.

"Que sea más receptiva, abierta, que me escuche más, si dices algo enseguida te corta"

"Que nos dejen hablar en la consulta. La hacen rápida y no te dejan hablar, no te da tiempo a hacer preguntas"

Otros muchos de los comentarios agrupados en la categoría de información hacían referencia a la necesidad de recibir una mayor cantidad y a una mayor calidad de esta, en forma oral y/o escrita:

"Que dé más información, más asertividad, que den explicaciones..."

"Me gustaría que me hablase, que me informara, pero no le puedes decir nada porque si no te toma coraje."

"Tener más información para los diabéticos porque se nos pueden escapar muchas cosas sobre cómo es la enfermedad, alimentación.., de lo que sólo tenemos conocimientos superficiales"

"Que te dieran por escrito el desarrollo de las curas, por si se te olvida algo ...informar" 
Una última categoría general amplia de comentarios hace alusión al deseo del paciente de que el profesional le dedique más tiempo y dedicación en la consulta, existiendo menos comentarios que hacen alusión a la demora en la atención:

"Simplemente más tiempo. Mi hija estuvo ingresada, ni la pesó, ni la midió..., porque estaba un poco alterada...Más paciencia."

"Que si vas más delicada que esté más tiempo, que te atiendan de otra forma. Que no tengan prisa cuando le vas explicando lo que te está pasando."

"Que escuche cuando le explico algo, más tiempo para atenderme, que esté pendiente de mí y no de otras cosas."

"Que dedicara más tiempo, por lo menos el suficiente para preguntar. En mi caso fue todo muy deprisa y se me olvidó preguntarle una cosa importante por las prisas."

\section{DISCUSIÓN}

Los hallazgos obtenidos en el presente estudio, en el que se compara en un cuestionario dos tipos de preguntas, abiertas y cerradas, la opinión directa e indirecta que tienen los usuarios sobre la actuación de los profesionales de enfermería que les atienden en los centros de salud, en cuatro dominios comunicativos: relación enfermera-paciente, información dada por la enfermera, tiempo empleado, procedimientos en la sala de curas y la toma de decisiones en la consulta de Enfermería, revelan algunos aspectos importantes de gran interés que pasamos a comentar.

En primer lugar desde el punto de vista cuantitativo llama la atención los altos niveles de satisfacción expresados por los pacientes en sus respuestas con respecto a las cuestiones planteadas, de forma cerrada, sobre alguno de lo dominios comunicativos (20), pero al preguntarle de manera abierta sobre el mismo dominio si tenían sugerencias para que la enfermera pudiera mejorar en el aspecto mencionado, un porcentaje significativo de ellos ofrecían propuestas concretas de deseos de cambio al respecto.

Podemos considerar que la existencia de estas "sugerencias de mejora" matiza el grado de satisfacción, al menos en aquellos pacientes que, en las preguntas cerradas, se declaraban satisfechos o muy satisfechos reduciendo este nivel de satisfacción inicial. Por tanto se plantea el hecho de que las encuestas de satisfacción directas ampliamente utilizadas en el entorno sanitario, inducen por su estructura de preguntas cerradas a respuestas que tienden a sobrevalorar el grado de satisfacción de los pacientes y que consecuentemente la introducción de preguntas abiertas inducen a plantear propuestas de optimización permitiendo detectar y visibilizar áreas de mejora concretas sobre los dominios explorados. Se han obtenido resultados análogos en otros estudios desarrollados con estrategias similares para ancianos residentes en instituciones ${ }^{(21-22)} \mathrm{O}$ en diferentes estrategias empleadas en el cuidado a pacientes crónicos ${ }^{(23)}$

Por otra parte desde el punto de vista descriptivo, el análisis cualitativo del estudio nos revela que la mayor proporción de discrepancias de respuesta, dada por los pacientes, las encontramos en el dominio referente al procedimiento en sala de curas. En dicho dominio encontramos categorías agrupadas con las proposiciones de 
mejora aportadas por los pacientes, estos sugerían en mayor proporción: la mejora de instalaciones e infraestructuras en la sala de curas que propicie más intimidad para el paciente; que se amplíe el espacio físico de la consulta y que se dote al profesional de material suficiente y adecuado para la cura, también sugieren más tiempo de cuidado y dedicación durante el proceso de la cura y, en menor proporción de respuestas, sugieren disminuir la demora en la atención.

Por otro lado, en el dominio de información recibida durante el proceso de atención en la consulta, encontramos categorías en las que los pacientes manifiestan la necesidad de ser más y mejor informados sobre todo de forma oral pero en algunos casos también por escrito, en aspectos específicos relacionados con el motivo de consulta programada o cura. En cuanto al dominio tiempo, las categorías agrupadas de las respuestas dadas por los pacientes hacen alusión explícitamente a la necesidad del paciente de ser atendido más y mejor por los profesionales de enfermería, tanto en las consultas programadas como en la sala de curas, en menor grado hacen alusión a la demora en las consultas. En los dominios referentes a la relación enfermerapaciente y toma de decisiones en la consulta de enfermería, las categorías de respuestas agrupadas hacen referencia a la necesidad del paciente de no recibir un trato frío, de que los profesionales los atiendan de forma personalizada, con simpatía y profesionalidad, de que los escuchen, de que les dediquen mas tiempo y de que los profesionales sean siempre los mismos, resultados en consonancia con la literatura existente al respecto ${ }^{(24)(25)}$.

Es importante hacer hincapié en la importancia de avanzar en el conocimiento de las necesidades en salud de los individuos, así como los factores que intervienen en la efectividad de las actividades sanitarias, y en particular de los propios profesionales, lo que hace posible prever un auge de la Investigación Cualitativa en campos como la Salud Pública y la Investigación de Servicios de Salud. Tal incremento debería acompañarse de una progresiva mejora en la calidad de la producción investigadora, para lo cual son necesarios instrumentos de evaluación de dicha calidad. Sin embargo, no hay que perder de vista que las implicaciones epistemológicas del enfoque cualitativo contribuyen a que la delimitación de dichos instrumentos haya sido objeto de controversia tanto a nivel conceptual como en el de sus métodos y técnicas ${ }^{(26)}$.

Por otro lado en cuanto a la percepción de la población en general de los diferentes servicios sanitarios, contamos con el estudio "Confianza en el Sistema Nacional de Salud" realizado en 2005 que refiere el aumento en la confianza de los españoles en el SNS gracias a la profesionalidad del personal sanitario, siendo los centros de Atención Primaria los más valorados, en clara coincidencia con Sindhu et al ${ }^{(27)}$ muy a pesar de las evidentes diferencias culturales y metodológicas de los dos estudios.

En la Encuesta de Satisfacción realizada por el Instituto de Estudios Sociales Avanzados del Consejo Superior de Investigaciones Científicas, en colaboración con el Servicio Andaluz de Salud (SAS) sobre la satisfacción del usuario de AP de $2008^{(7)}$ se comprueba que los usuarios de los servicios sanitarios públicos en Andalucía valoran a los diferentes profesionales del SAS, con niveles de satisfacción por encima del $85 \%$, siendo los mejor valorados los profesionales de enfermería con un $94,2 \%$, seguido de los médicos/pediatras (93,2\%), los celadores en un $87,4 \%$, y los auxiliares de enfermería en último lugar con un $82,3 \%$.

Otros estudios de satisfacción y calidad percibida en diferentes ámbitos sanitarios, concluyen que los aspectos mejor valorados son el trato de respeto y la información 
recibida por los profesionales, así como los conocimientos técnicos ${ }^{(20)}$. Por otra parte, un $94 \%$ de pacientes encuestados esperan recibir un trato aceptable, valorando esta amabilidad por encima de componentes científico-técnicos ${ }^{(2)}$. Estos datos muestran al aspecto comunicacional como el más relevante, Boscart VM ${ }^{(28)}$ en su estudio refleja los resultados positivos en la relación enfermera paciente posterior a un programa educacional con las enfermeras en aspectos relacionales; aspecto este corroborado en otros estudios como el de González Valentin, et al ${ }^{(29)}$ en el que concluye sugiriendo actualizar a las enfermeras en habilidades de comunicación como medio de modificar factores que intervienen en la mejor satisfacción del paciente.

En este apartado se han hecho diferentes consideraciones a partir del aspecto relacional enfermera paciente y su influencia en la satisfacción de este último. Lo que conduce a considerar que la mejora en ese aspecto relacional, no solo influiría en la satisfacción del paciente sino también en una de las funciones fundamentales en el ámbito de la Enfermería de Atención Primaria, La Educación para la Salud ${ }^{(30)}$

\section{CONCLUSIÓN}

La interpretación que hacemos de los resultados del estudio es que la mayoría de los pacientes que acuden a las consultas de enfermería, en los centros de salud, muestran satisfacción con la atención recibida, si bien les gustaría participar más en la toma de decisiones. Las sugerencias de los pacientes recogidas en este trabajo nos están mostrando líneas para diseñar programas formativos dirigidos a los profesionales de enfermería con el objetivo de adecuar la implicación de los pacientes en la toma de decisiones en las consultas de manera más adecuada, así como intervenciones concretas de carácter organizativo a realizar por planificadores sanitarios, en aquellos aspectos relacionados con cambios de personal o incluso tiempos de consulta

\section{REFERENCIAS BIBLIOGRÁFICAS}

1.- Contrato Programa 2010-2013. Consejería de Salud. Servicio Andaluz de Salud. Junta de Andalucía.

2 - Magro Perteguer R. Expectativas y satisfacción de los usuarios de Atención Primaria. Semergen. 1998; 24 (9): 711-718

3 - Donabedian. La calidad de la atención médica. A. La prensa mexicana. México 1984

4.- Heather Palmer R. Evaluación de la asistencia ambulatoria. Principios y Prácticas. Ministerio de Sanidad y Consumo. Madrid 1989

5.- La mesura de la satisfacció en els serveis sanitario. Gac. Sanitaria 1992; 6: 178185

6.- Díaz R. La Calidad percibida en la Sanidad Pública. Revista de Calidad Asistencial 2005; 20 (1): 35-42

7.- Serrano del Moral R, Ranchal Romero J. La importancia e invisibilidad de las enfermeras en Atención Primaria de Salud. Index Enfermería 2010; 19 (1). Disponible en:

http://scielo.isciii.es/scielo.php?pid=S113212962010000100002\&script=sci arttext (Consultado el día 14/07/12)

8.- Pujiula Masó J, Suñer Soler R, Puisdemont Guinart M, Grau Martín A. Bertran Noguer C. Hortal Gasul G et al. La satisfacción de los pacientes hospitalizados como indicador de la calidad asistencial. Enfermería Clínica 2006; 16 (1):19-26 
9.- Prieto Rodríguez M A, March Cerda J C, López Fernández L A. Calidad percibida por usuarios de centros de salud y aseguradoras privadas.; Escuela Andaluza de Salud Pública. Atención Primaria. 1999; 24 (5):259-66

10.- Encuesta de satisfacción de los usuarios con los servicios de Atención Primaria. Madrid INSALUD, 2002

11 Asenjo M A. El Hospital como empresa. Medic Clínica (Barc.) 1991; 96:780-3

12.- Vázquez Jiménez, M.; Araque López MD; Díaz Suárez M; Jiménez Alaminos M; Izquierdo Muñoz AB. Evaluación de la percepción de la calidad asistencial de enfermería por el paciente ingresado en una Unidad Hospitalaria-; Servicio de Medicina Interna. Hospital General de la Mancha. Alcazar de San Juan. Disponible en: http://directivos.publicacionmedica.com/spip.php?article59 (Consultado el día 14/07/12)

13.- Rosa María García Pardo. Calidad percibida por los usuarios de las consultas de enfermería de A.P. Diferencias en la percepción de la población inmigrante respecto a la población autóctona. Reduca. Serie de Trabajos Fin de Master. 2009; 1(2): 913-926 14.- Delgado Valencia AM. Álvarez Rodríguez Y, Aliaga Guerra RM. Calidad de Atención de Enfermería desde la percepción del usuario que acude al servicio de emergencia del Hospital Nacional Guillermo Almenara Irigoyen. Rev. Ciencias de la Salud 2007; 2:2

15.- González Valentín A, Padin López S, De Ramón Garrido, E. Patient Satisfaction With Nursing Care in a Regional University Hospital in Southern Spain. Journal of Nursing Care Quality 2005; 20 (1):63-72

16.- Blanco Abril S, Sánchez Vicario F, Chinchilla Nevado MA, Cobrero Jiménez LM, Mediavilla Durango M, Rodríguez Gonzalo A, Cuñago Barrio A. Satisfacción de los pacientes de urgencias con los cuidados enfermeros. Enfermería Clínica 2010; 20(1):23-31

17.- Ángel Luis Olivera Escalona. Satisfacción percibida por los pacientes atendidos en el servicio de urgencias. Gestión sanitaria. Medicina de Urgencias. Publicado el 287-2009

18.- De la Fuente Rodríguez, A.; Fernández Lerones, MJ; Hoyos Valencia, Y; León Rodríguez, C; Zuloaga Mendiolea, C; Ruiz Garrido, M: Servicio de Urgencias. Estudio de calidad percibida y satisfacción de los usuarios de la Zona Básica de salud Altamira. Rev. de calidad Asistencial 24 (3):109-14-2009

19.- A.B. Meseguer Henarejos. Rev. Iberoamericana de Fisioterapia y Kenisiologia. Calidad percibida en la fisioterapia de Atención Primaria. Situación y oportunidades de mejora en la cervicalgia. 2008, 11(2):68-80

20.- Servicio Andaluz de Salud; 2006 [sede Web]. Sevilla: Junta de Andalucía [acceso 15 de septiembre de 2010]. Cuestionario de satisfacción Atención Primaria SAS.

21.- Levy-Storms L, Simmons SF, Gutierrez VF, Miller-Martínez D, Hickey K, Schenelle JF. A refined protocol for coding nursing home residents comments during satisfaction interviews. J Gerontology Social Sciences 2005; 60B: S326-S330.

22. - Levy-Storms L, Schenelle JF, Simmons SF. A comparison of methods to assess nursing home residents unmet needs. The Gerontologist 2002; 42: 454-61.

23.- Wakefield BJ, Holman JE, Ray A, Scherubel M . Patient perceptions of a remote monitoring intervention for chronic disease management Journal of Gerontological Nursing 2011; 37(4):16-20. Disponible en: http://www.ncbi.nlm.nih.gov/pubmed/21417194 (consultado el día 2/7/2012)

24.- Mahomed R, St John W, Patterson E. Understanding the process of patient satisfaction with nurse-led chronic disease management in general practice. Journal of Advanced Nursing. 2012; doi: 10.1111/j.1365-2648.2012.05953.x. Disponible en: http://www.ncbi.nlm.nih.gov/pubmed/22384855 (consultado el día 2/7/2012) 
25.- Hernández Meca M E, Ochando García A, Mora Canales J, Lorenzo Martínez S, López Revuelta K. Satisfacción del paciente en una unidad de hemodiálisis: Objetivo de calidad asistencial en enfermería. Rev Soc Esp Enferm Nefrol [revista en Internet]. 2005 Jun [citado 2012 Jul 14]; 8(2): 90-96

Disponible en:

http://scielo.isciii.es/scielo.php?script=sci_arttext\&pid=S113913752005000200002\&lng $=$ es.

26.- Calderón, C. Criterios de calidad en la Investigación Cualitativa en Salud (ICS): Apuntes para un debate necesario. Revista Española de Salud Pública, 2002; 76: 473-482

27.- Sindhu S, Pholpet C, Puttapitukpol S. Meeting the challenges of chronic illness: a nurse-led collaborative community care program in Thailand. Collegian $2010 ; 17$ (2): 93-99 Disponible en: http://www.ncbi.nlm.nih.gov/pubmed/20738062 (consultado el día 2/7/2012)

28.- Boscart V. M. A communication intervention for nursing staff in chronic care. Journal of Advanced Nursing 2009; 65: 1823-1832. Disponible en: http://www.ncbi.nlm.nih.gov/pubmed/19694845 (consultado el día 2/7/2012)

29.- González-Valentín MA, Padín López S, De Ramón Garrido E. Satisfacción del paciente con la atención de enfermería. Enferm Clin. 2005; 15 (3): 147-55. Disponible en:

http://www.elsevier.es/sites/default/files/elsevier/pdf/35/35v15n03a13075772pdf001.pd f (consultado el día 14/7/2012)

30.- Orta González MA, Rodríguez Muñiz RM, Prieto Tinoco J. Revista ROL de enfermería 2007; 30 (4):15-17 


\section{Código:}

Nos gustaría que a continuación nos contestara algunas preguntas sobre algunos aspectos relacionados con la visita que ha hecho a la enfermera:

\section{RELACIÓN ENFERMERA-PACIENTE}

1.1.- ¿Cómo de satisfecho/a se sintió con la forma en la que la enfermera se comportó con usted ese día en la consulta?
( ) Muy satisfecho-a
( ) Satisfecho-a
( ) Regular de satisfecho-a
( ) Insatisfecho-a

1.2.- ¿Cree usted que la enfermera fue suficientemente comprensiva y amable hacia su situación/problema?

1.3.- Si usted pudiera cambiar algo de la forma en que la enfermera le trató ¿Qué cambiaria para mejorar ese trato en el futuro?

\section{2-INFORMACIÓN}

2.1.- ¿Cómo de satisfecho/a está con la forma en la que la enfermera le informó sobre lo que se puede hacer para mejorar su problema?

(Por ejemplo, el tratamiento que debe de seguir, la forma en la que usted puede hacerlo o cuidarse usted mismo, los efectos $2^{\circ} \circ$ complicaciones que pueden aparecer, y como tiene usted que actuar...).
( ) Muy satisfecho/a
( ) Satisfecho/a
( ) Regular de satisfecho/a
( ) Insatisfecho/a 
2.2.- ¿Considera suficiente la información que le dio la enfermera sobre el problema que usted tenía?

2.3.- Si usted pudiera cambiar algo de la forma en la que la enfermera le informó ¿Qué sería lo que cambiaría para mejorar en el futuro?

\section{3-TIEMPO EMPLEADO}

3.1.- ¿Cómo de satisfecho/a está con el tiempo que la enfermera le dedicó a usted durante su consulta?
( ) Muy satisfecho/a
( ) Satisfecho/a
( ) Regular de satisfecho/a
( ) Insatisfecho/a

3.2.- En relación al tiempo que le dedicó la enfermera ¿Lo considera adecuado o le hubiera gustado que hubiera sido menor o mayor?

3.3.- Si usted pudiera cambiar algo respecto al tiempo que empleó la enfermera al realizar su trabajo ¿Qué cambiaría para mejorar en el futuro?

\section{4-PROCEDIMIENTOS EN LA SALA DE CURAS. (Anotar si procede)}

4.1.- ¿Cómo de satisfecho/a está usted con la forma en que la enfermera realizó la tarea o procedimiento por el que usted acudió a la sala de curas? (Aplicable a: curas de heridas, retirada de suturas) 
( ) Muy satisfecho/a

( ) Satisfecho/a

() Regular de satisfecho/a

( ) Insatisfecho/a

4.2.- Con respecto al problema que le llevó a la sala de curas ¿le habría gustado que la enfermera le hubiese atendido en algo más?

4.3.- Si pudiera cambiar algo de la forma en la que la enfermera le llevó a cabo el procedimiento ¿Qué sería lo que cambiaría o sugeriría para que se realizara mejor en el futuro?

(Aplicable a: curas de heridas, retirada de suturas)

\section{5-TOMA DE DECISIONES EN LA CONSULTA DE ENFERMERIA.} (Anotar si procede)

Usted sabe que cuando acude a la consulta de enfermería por un problema de salud, en ocasiones, la enfermera y usted deben tomar una serie de decisiones sobre recomendaciones adecuadas para su salud (cambio de hábitos como ejercicio, dieta, alcohol, tabaco, y/o seguir o no tratamiento prescrito):

5.1.- ¿Cómo de satisfecho está con la forma en la que se decidió lo que tenia que hacer para mejorar o solucionar el problema por el que acudió aquel día a la consulta de enfermería?
( ) Muy satisfecho/a
() Satisfecho/a
( ) Regular de satisfecho/a
( ) Insatisfecho/a

5.2.a.- A veces existen varias recomendaciones igualmente validas y eficaces de tratar sus problemas de salud (varias opciones). Cuando esto sucede ¿Quién cree usted que es el que debe decidir lo que hay que hacer?

( ) La enfermera es quien debe decidir siempre lo que el paciente debe hacer

( ) Lo deben decidir de mutuo acuerdo la enfermera y el paciente

( ) Sólo yo soy quien debe decidir lo que hacer 
5.2b.- En la consulta en la que se trató lo que se podía hacer respecto a su problema (por ejemplo cambiar de hábitos y/o seguir o no tratamiento). ¿Participó usted en la decisión que finalmente se tomó?

\begin{tabular}{|c|c|c|c|c|}
\hline $\begin{array}{l}\mathbf{0} \\
\text { NO PARTICIPÉ } \\
\text { EN ABSOLUTO } \\
\text { (FUE LA ENFERMERA LA } \\
\text { QUE DECIDIO LO } \\
\text { QUE TENÍA QUE HACER) }\end{array}$ & 3 & $\begin{array}{ccc}4 & \mathbf{5} & 6 \\
\text { LO DECIDIMOS ENTRE } \\
\text { LA ENFERMERA Y YO }\end{array}$ & 7 & $\begin{array}{rcr}8 & \mathbf{1 0} \\
\text { PARTICIPÉ } \\
\text { COMPLETAMENTE } \\
\text { (FUI YO EL QUE } \\
\text { INFORMADO POR LA } \\
\text { ERMERA DECIDIO QUE } \\
\text { HACER) }\end{array}$ \\
\hline
\end{tabular}

5.2c.- ¿Le hubiera gustado participar mas en la decisión que se tomo sobre lo que tiene usted que hacer para tratar su problema de salud?

5.3.- ¿Qué le diría a su enfermera para que en un futuro le ayude a participar en las decisiones que hay que tomar en la consulta de enfermería y que le atañen a usted directamente? 